\title{
Density Autocorrelation Function in a Classical Fluid from Initial Correlations*
}

\author{
F. Lado \\ Department of Physics, North Carolina State University, Raleigh, North Carolina 27607 \\ (Received 16 March 1970)
}

\begin{abstract}
A complete set of time-independent orthogonal phase functions $\left\{\Psi_{s}\right\}, s=0,1,2, \ldots$, is generated via the Schmidt process and used to represent the Fourier coefficient $R_{\overrightarrow{\mathbf{k}}}(t)$ of the time-dependent microscopic density function. The projection of $R_{\overrightarrow{\mathrm{k}}}(t)$ on $\Psi_{0}$ is essentially the density autocorrelation function. The equation of motion of the coefficients of this expansion is found and formally solved to yield the Laplace-Fourier transform of the density autocorrelation function as a ratio of infinite determinants, closely related to Mori's continued-fraction expansion. A non-Markovian memory function is then readily defined in the same terms. These exact results are illustrated by explicit calculations for the ideal gas. Finally, a perturbation expansion of the memory function is developed, leading to practical approximations.
\end{abstract}

\section{INTRODUCTION}

The simultaneous correlation between the particle density at two points $\vec{r}^{\prime}$ and $\vec{r}^{\prime}+\vec{r}$ in an equilibrium fluid, given by the pair distribution function $g(\vec{r})$, is a fundamental property of the fluid from which other properties may be obtained. The calculation of this quantity is a statistical problem in which much progress has been made in recent years. When the density at the second point is taken at a time $t$ later than the first, however, an additional many-body dynamical calculation is required to obtain the corresponding time-dependent correlation function $G(\overrightarrow{\mathrm{r}}, t)$, first introduced by Van Hove. ${ }^{1}$ This is, of course, an initial-value problem, so that $G(\overrightarrow{\mathrm{r}}, t)$ should be expressible in terms of the state of the system at the initial time $t=0$. In practice, however, this is a difficult problem that is often partially circumvented by physically motivated assumptions.

An elegant approach to the calculation of timecorrelation functions such as $G(\overrightarrow{\mathrm{r}}, t)$ has been given by Zwanzig, ${ }^{2}$ who used a projection-operator technique to obtain an equation of motion involving a non-Markovian memory function. A formal definition of this function is obtained which of course then contains all of the original dynamical problems of the calculation. The present work was motivated by a desire to make this projection technique more explicit, in the hopes of thereby facilitating approximations to the functional dependence of the memory function.

The general formalism is developed in Sec. II. ${ }^{3}$ Here the Fourier coefficients of the density at time $t$ are expanded onto a set of orthogonal functions generated by the Schmidt process. The coefficients of this expansion are time-correlation functions, and in particular the first coefficient, i. e., the explicit projection of the total density function onto the first or thogonal function, is just the autocorrelation function being sought. The Laplace transform of this quantity [essentially the doubly transformed $G(\overrightarrow{\mathbf{r}}, t)]$ is then resolved as a ratio of infinite-order determinants, the elements of which contain only correlations from the initial state at $t=0$, thus formally satisfying the rigorous requirements of an initial-value problem. A memory function is then readily defined in the same terms. These general results are illustrated in Sec. III by applying them to the ideal gas, where explicit evaluations can be made. In Sec. IV a practical approximation to the memory function is obtained using a perturbation expansion.

After this work was completed, the author found that a general analysis of time-correlation functions similar to that in Sec. II had been made by Mori. ${ }^{4}$ The form of the final result, however, was that of a continued fraction, rather than a ratio of determinants, as below. One of the advantages of the present formulation is that the final result lies in the range of well-studied functions, so that a body of known results immediately becomes available for further developments (as instanced, e.g., in the perturbation expansion of Sec. IV).

\section{GENERAL FORMALISM}

\section{Preliminaries}

The system consists of $N$ molecules, in an equilibrium distribution in a volume $V$ at a temperature $T=\left(k_{B} \beta\right)^{-1}$, whose motion is governed by the Hamiltonian

$$
H=\sum_{j=1}^{N} \frac{\overrightarrow{\mathrm{p}}_{j}^{2}}{2 m}+U\left(\overrightarrow{\mathrm{r}}_{1}, \cdots, \overrightarrow{\mathrm{r}}_{N}\right) .
$$

The microscopic particle density at some arbitrarily chosen origin of time is denoted

$$
\rho(\overrightarrow{\mathrm{r}}, 0)=\sum_{j=1}^{N} \delta\left(\overrightarrow{\mathrm{r}}-\overrightarrow{\mathrm{r}}_{j}\right) .
$$


This function evolves in time according to the classical equation of motion

$$
\frac{\partial \rho(\overrightarrow{\mathrm{r}}, t)}{\partial t}=-[H, \rho(\overrightarrow{\mathrm{r}}, t)] \equiv i L \rho(\overrightarrow{\mathrm{r}}, t),
$$

whose formal solution is

$$
\rho(\overrightarrow{\mathrm{r}}, t)=e^{i t L \rho}(\overrightarrow{\mathrm{r}}, 0) \text {. }
$$

Here $L$ is the (Hermitian) Liouville operator, defined as indicated by $i$ times the Poisson bracket of $H$ with the operand. The mean value of the density at any time is $n \equiv N / V$ and we define for convenience the modified density function

$$
R(\overrightarrow{\mathrm{r}}, t)=\rho(\overrightarrow{\mathrm{r}}, t)-n,
$$

whose mean is zero.

The main quantity of interest in this paper is the space- and time-dependent density correlation function $G(\overrightarrow{\mathrm{r}}, t)$ defined by ${ }^{1,5}$

$$
G(\overrightarrow{\mathrm{r}}, t)=(n N)^{-1} \int d \overrightarrow{\mathrm{r}}^{\prime}\left\langle R\left(\overrightarrow{\mathrm{r}}^{\prime}, 0\right) R\left(\overrightarrow{\mathrm{r}}^{\prime}+\overrightarrow{\mathrm{r}}, t\right)\right\rangle,
$$

where the brackets denote a canonical-ensemble average. Note that initially

$$
G(\overrightarrow{\mathrm{r}}, 0)=G(\overrightarrow{\mathrm{r}})+n^{-1} \delta(\overrightarrow{\mathrm{r}}),
$$

where

$$
G(\overrightarrow{\mathrm{r}}) \equiv g(\overrightarrow{\mathrm{r}})-1
$$

is the total correlation function of equilibrium fluids. The subsequent analysis will actually be made in terms of the Fourier coefficients of $G(\overrightarrow{\mathrm{r}}, t)$, namely,

$$
G_{\overrightarrow{\mathrm{k}}}(t)=(n N)^{-1}\left\langle R_{\overrightarrow{\mathrm{k}}}^{*}(0) R_{\overrightarrow{\mathrm{k}}}(t)\right\rangle,
$$

where

$$
\begin{aligned}
& R_{\overrightarrow{\mathrm{k}}}(t)=e^{i t L} R_{\overrightarrow{\mathrm{k}}}(0) \\
& R_{\overrightarrow{\mathrm{k}}}(0)=\sum_{j=1}^{N} e^{-i \overrightarrow{\mathrm{k}} \cdot \overrightarrow{\mathrm{r}}_{j}}-N \delta_{\overrightarrow{\mathrm{k}}, 0} .
\end{aligned}
$$

In general, for a function $F(\vec{r})$ defined in the volume $V$, we write the Fourier expansion in the form

$$
\begin{aligned}
F(\overrightarrow{\mathrm{r}}) & =V^{-1} \sum_{\overrightarrow{\mathrm{k}}} F_{\overrightarrow{\mathrm{k}}} e^{i \overrightarrow{\mathrm{k}} \cdot \overrightarrow{\mathrm{r}}}, \\
F_{\overrightarrow{\mathrm{k}}} & =\int d \overrightarrow{\mathrm{r}} F(\overrightarrow{\mathrm{r}}) e^{-i \overrightarrow{\mathrm{k}} \cdot \overrightarrow{\mathrm{r}}} .
\end{aligned}
$$

In Eq. (10b), $\delta_{\vec{k}, 0}$ is the Kronecker $\delta$.

$$
\text { Expansion of } R_{\overrightarrow{\mathrm{z}}}(t)
$$

The dynamical problem is contained in the calculation of $R_{\overrightarrow{\mathrm{k}}}(t)$. An expansion of this quantity onto a basis set of time-independent phase functions may be trivially obtained by expanding the exponential operator in Eq. (10a), which gives

$$
R_{\overrightarrow{\mathrm{k}}}(t)=\sum_{s=0}^{\infty} \frac{(i t)^{s}}{s !} L^{s} R_{\overrightarrow{\mathrm{k}}}(0)
$$

This straightforward time expansion, however, leads to a slowly converging series for the timecorrelation function, with, what is worse, coefficients that increase rapidly in complexity. The set of functions $\left\{L^{s} R_{\overrightarrow{\mathrm{k}}}(0)\right\}, s=0,1,2, \ldots$ may, however, be used to generate an orthogonal set of basis functions $\left\{\Psi_{s}\right\}, s=0,1,2, \ldots$, which, being tailored to the particular dynamics of the problem at hand, have many convenient properties. (The $\overrightarrow{\mathrm{k}}$ dependence of the $\Psi_{s}$, and of several other quantities in the following, will be suppressed in the notation. ) We use the Schmidt orthogonalization process and define

$$
\begin{aligned}
& \Psi_{0}=R_{\vec{k}}(0), \\
& \Psi_{s}=L^{s} R_{\overrightarrow{\mathrm{k}}}(0)-\sum_{n=0}^{s-1} \frac{\left\langle\Psi_{n}^{*} L^{s} R_{\vec{k}}(0)\right\rangle}{\left\langle\Psi_{n}^{*} \Psi_{n}\right\rangle} \Psi_{n}, \quad s>0 .
\end{aligned}
$$

By construction then these functions have the property that

$$
\left\langle\Psi_{s}^{*} \Psi_{n}\right\rangle=\left\langle\Psi_{s}^{*} \Psi_{s}\right\rangle \delta_{s n} .
$$

(Note that the $\Psi_{s}$ are being left unnormalized.) In terms of this orthogonal set, we may now write

$$
R_{\overrightarrow{\mathrm{k}}}(t)=\sum_{s=0}^{\infty} A_{s}(t) \Psi_{s},
$$

where

$$
A_{s}(t)=\left\langle\Psi_{s}^{*} R_{\vec{k}}(t)\right\rangle /\left\langle\Psi_{s}^{*} \Psi_{s}\right\rangle .
$$

One finds then that the coefficients $A_{s}(t)$ are themselves time-correlation functions, and in particular that

$$
A_{0}(t)=\frac{\left\langle\Psi_{0}^{*} R_{\overrightarrow{\mathrm{k}}}(t)\right\rangle}{\left\langle\Psi_{0}^{*} \Psi_{0}\right\rangle} \equiv \frac{G_{\overrightarrow{\mathrm{k}}}(t)}{G_{\overrightarrow{\mathrm{k}}}(0)} .
$$

That is, in order to calculate the autocorrelation function $G_{\overrightarrow{\mathrm{k}}}(t)$ we need to know only the projection of the total "vector" $R_{\overrightarrow{\mathrm{k}}}(t)$ onto the fixed basis "vector" $\Psi_{0}$.

Note finally that since $R_{\overrightarrow{\mathrm{k}}}(0)=\Psi_{0}$, the initial values of the $A_{s}(t)$ coefficients are

$$
A_{0}(0)=1, A_{s}(0)=0, s>0 .
$$

Equation of Motion of the $A_{s}(t)$

The equation for the evolution of the $A_{s}(t)$ may be readily obtained. From Eq. (10a) we have

$$
\frac{\partial R_{\overrightarrow{\mathrm{k}}}(t)}{\partial t}=i L R_{\overrightarrow{\mathrm{k}}}(t)
$$

and hence, using (14) and (15), we get

$$
\frac{\partial A_{s}(t)}{\partial t}=i \sum_{n=0}^{\infty} A_{n}(t) \frac{\left\langle\Psi_{s}^{*} L \Psi_{n}\right\rangle}{\left\langle\Psi_{s}^{*} \Psi_{s}\right\rangle} .
$$

It is shown in Appendix A that the matrix whose elements appear in this equation has a very simple form, namely, 


$$
\frac{\left\langle\Psi_{s}^{*} L \Psi_{n}\right\rangle}{\left\langle\Psi_{s}^{*} \Psi_{s}\right\rangle}=\delta_{s, n+1}+\nu_{s} \delta_{s, n-1}
$$

where

$$
\nu_{s}=\frac{\left\langle\Psi_{s+1}^{*} \Psi_{s+1}\right\rangle}{\left\langle\Psi_{s}^{*} \Psi_{s}\right\rangle} .
$$

Thus Eq. (20) reduces to a simple relation between any three successive coefficients $A_{s}(t)$ (except for $s=0$ as shown):

$$
\begin{aligned}
& \frac{-i \partial A_{0}(t)}{\partial t}=\nu_{0} A_{1}(t), \\
& \frac{-i \partial A_{s}(t)}{\partial t}=A_{s-1}(t)+\nu_{s} A_{s+1}(t), \quad s>0 .
\end{aligned}
$$

This set of linear differential equations may be conveniently solved (at least formally) in Laplacetransform representation. We will denote the Laplace transform of a function by its script letter. The transformed Eqs. (23) then yield the set of algebraic equations

$$
\begin{aligned}
i z a_{0}(z)+\nu_{0} a_{1}(z) & =i, \\
a_{0}(z)+i z Q_{1}(z)+\nu_{1} a_{2}(z) & =0, \\
a_{1}(z)+i z a_{2}(z)+\nu_{2} a_{3}(z) & =0,
\end{aligned}
$$

where we have used Eqs. (18) in the right-hand side. A straightforward application of Cramer's rule then gives the solution (see comments in the next paragraph)

$$
a_{0}(z)=i D_{1}(z) / D_{0}(z),
$$

where $D_{s}(z)$ is an infinite-order determinant,

$$
D_{s}(z)=\left|\begin{array}{ccccc}
i z & \nu_{s} & 0 & 0 & \cdots \\
1 & i z & \nu_{s+1} & 0 & \cdots \\
0 & 1 & i z & v_{s+2} & \cdots \\
0 & 0 & 1 & i z & \cdots \\
\vdots & \vdots & \vdots & \vdots & \ddots
\end{array}\right|,
$$

having nonzero elements only on the principal diagonal and the two immediately adjacent diagonals, as shown.

The ratio of two infinite-order determinants such as appears in Eq. (25) must clearly be defined by a limiting process. This may be done unambiguously by first terminating the original expansion of $R_{\overrightarrow{\mathrm{k}}}(t)$ [Eq. (15)] at finite $s=S$, proceeding through the above solution, and finally taking the limit as $S \rightarrow \infty$. For finite $S$ this yields for $\alpha_{0}(z)$ the solution $i D_{1}^{(S)}(z) / D_{0}^{(S)}(z)$, where now

$$
D_{n}^{(s)}(z)=\left|\begin{array}{llllll}
i z & \nu_{n} & 0 & \cdots & 0 & 0 \\
1 & i z & \nu_{n+1} & \cdots & 0 & 0 \\
0 & 1 & i z & \cdots & 0 & 0 \\
\vdots & \vdots & \vdots & \ddots & \vdots & \vdots \\
0 & 0 & 0 & \cdots & i z & \nu_{s-1} \\
0 & 0 & 0 & \cdots & 1 & i z
\end{array}\right| .
$$

We then define

$$
D_{1}(z) / D_{0}(z)=\lim _{S \rightarrow \infty}\left[D_{1}^{(S)}(z) / D_{0}^{(S)}(z)\right] .
$$

Note that for any finite $S$ the denominator in (28) is a polynomial in $z$ of one degree higher than the numerator. Other ratios of infinite-order determinants will also be understood in the sense of the limiting process in Eq. (28).

Noting that $A_{0}(t)$ is essentially the density autocorrelation function [Eq. (17)] and that the determinants $\mathfrak{D}_{n}(z)$ contain only correlations at the initial time [Eq. (22) ], we may say that Eq. (25) represents the desired solution of the time evolution of $G(\vec{r}, t)$ in terms of the state of the system at time $t=0$. This of course is true only in a formal sense, for the problem of evaluating the determinants and performing the Laplace inversion of (25) is clearly impossible for the general interacting case where the coefficients $\nu_{s}$ will largely remain unknown. Rather than seek an approximate inversion of Eq. (25), however, we will in the following subsection introduce a "memory function" for the evolution of $G(\overrightarrow{\mathrm{r}}, t)$ and later, in Sec. IV, seek to approximate it.

\section{Memory Function}

By expanding $D_{0}(z)$ in minors of the first row (or column) we may write Eq. (25) in the form

$$
\begin{aligned}
a_{0}(z) & =i D_{1}(z) /\left[i z D_{1}(z)-\nu_{0} D_{2}(z)\right] \\
& =\left[z+i \nu_{0} D_{2}(z) / D_{1}(z)\right]^{-1}=\left[z+\mathcal{K}_{\overrightarrow{\mathrm{k}}}(z)\right]^{-1},
\end{aligned}
$$

where we have defined (reintroducing the subscript $\overrightarrow{\mathrm{k}}$ notation)

$$
\mathfrak{K}_{\overrightarrow{\mathrm{k}}}(z) \equiv i \nu_{0} \mathfrak{D}_{2}(z) / \mathfrak{D}_{1}(z) .
$$

The role of this quantity becomes evident on rewriting Eq. (29) in the form

$$
z Q_{0}(z)-1=-\kappa_{\overrightarrow{\mathrm{x}}}(z) Q_{0}(z)
$$

and performing the Laplace inversion; this yields

$$
\frac{\partial A_{0}(t)}{\partial t}=-\int_{0}^{t} d t^{\prime} K_{\overrightarrow{\mathrm{k}}}\left(t-t^{\prime}\right) A_{0}\left(t^{\prime}\right),
$$

or, using (17),

$$
\frac{\partial G_{\overrightarrow{\mathrm{k}}}(t)}{\partial t}=-\int_{0}^{t} d t^{\prime} K_{\overrightarrow{\mathrm{k}}}\left(t-t^{\prime}\right) G_{\overrightarrow{\mathrm{k}}}\left(t^{\prime}\right) .
$$

That is, $K_{\overrightarrow{\mathbf{k}}}(t)$ is a non-Markovian memory function for the evolution of $G_{\overrightarrow{\mathrm{k}}}(t) .^{2}$

The initial value of this function may be found from the asymptotic limit of $\mathcal{K}_{\overrightarrow{\mathrm{k}}}(z)$. Noting in Eq. (30) that the denominator is again of degree one higher than the numerator, we obtain for this limit simply

$$
\varkappa_{\overrightarrow{\mathrm{k}}}(z) \sim \nu_{0} / z, \quad z \rightarrow \infty
$$


so that

$$
\varkappa_{\overrightarrow{\mathrm{k}}}(0)=\nu_{0}=\left(k^{2} / \beta m\right)\left(1-n C_{\overrightarrow{\mathrm{k}}}\right),
$$

where the last equality is from Appendix B. Here $C_{\overrightarrow{\mathrm{k}}}$ is the Fourier coefficient of the direct correlation function $C(\vec{r})$ of classical equilibrium fluids.

If we define a time-dependent function $C_{\overrightarrow{\mathrm{k}}}(t)$ such that

$$
K_{\overrightarrow{\mathrm{k}}}(t)=\left(n k^{2} / \beta m\right) C_{\overrightarrow{\mathrm{k}}}(t),
$$

then Eq. (33) becomes

$$
\frac{\partial G_{\overrightarrow{\mathrm{k}}}(t)}{\partial t}=\frac{n k^{2}}{\beta m} \int_{0}^{t} d t^{\prime} C_{\overrightarrow{\mathrm{k}}}\left(t-t^{\prime}\right) G_{\overrightarrow{\mathrm{k}}}\left(t^{\prime}\right),
$$

or, by Fourier inversion,

$$
\begin{aligned}
\frac{\partial G(\overrightarrow{\mathrm{r}}, t)}{\partial t}= & -\frac{n}{\beta m} \nabla^{2} \int_{0}^{t} d t^{\prime} \int d \overrightarrow{\mathrm{r}}^{\prime} \\
& \times C\left(\overrightarrow{\mathrm{r}}-\overrightarrow{\mathrm{r}}^{\prime}, t-t^{\prime}\right) G\left(\overrightarrow{\mathrm{r}}^{\prime}, t^{\prime}\right) .
\end{aligned}
$$

This is just the defining equation suggested by Percus and Yevick ${ }^{6}$ for a generalized, space- and timedependent direct correlation function $C(\vec{r}, t)$.

We note finally that by indefinite continuation of the process of expanding the determinant in the denominator, as begun in Eq. (29), the continuedfraction expansion of $\mathrm{Mori}^{4}$ may be recovered.

\section{NONINTERACTING CASE}

For the sake both of illustrating the developments of Sec. II and of obtaining formulas needed below, we will display here explicit evaluations of the general quantities introduced above for the case of the noninteracting gas, $U=0$.

The realization of the program set forth in Sec. II hinges on a knowledge of the coefficients $\nu_{s}$. For the present simple case, it is shown in Appendix B that

$$
\nu_{s}^{\circ}=(s+1) k^{2} / \beta m, \quad s=0,1,2, \cdots
$$

where the superscript degree mark identifies this ideal case, and furthermore that

$$
\begin{aligned}
\Psi_{s}^{\circ}= & (-1)^{s}\left(\frac{k^{2}}{\beta m}\right)^{s / 2} \sum_{j=1}^{N} e^{-i \overrightarrow{\mathrm{k}} \cdot \overrightarrow{\mathrm{r}}_{j}} \\
& \times H_{s}\left[\left(\frac{\beta}{m}\right)^{1 / 2} \hat{k} \cdot \overrightarrow{\mathrm{p}}_{j}\right]-N \delta_{\overrightarrow{\mathrm{k}}, 0} \delta_{s, 0},
\end{aligned}
$$

where the $H_{s}(x)$ are Hermite polynomials and $\hat{k}$ is a unit vector in the direction of $\vec{k}$. In view of the fact that the canonical distribution function contributes a Gaussian weight function for the momenta, it is not surprising that the orthogonal expansion should in this case turn out to depend essentially on Hermite polynomials.

The corresponding coefficients $A_{s}^{\circ}(t)$ must satisfy Eqs. (23), which now read

$$
\begin{aligned}
& -i \frac{\partial A_{0}^{\circ}(t)}{\partial t}=\frac{k^{2}}{\beta m} A_{1}^{\circ}(t) \\
& -i \frac{\partial A_{s}^{\circ}(t)}{\partial t}=A_{s-1}^{\circ}(t)+(s+1) \frac{k^{2}}{\beta m} A_{s+1}^{\circ}(t), \quad s>0
\end{aligned}
$$

along with the initial conditions of Eqs. (18). It can be verified immediately that the solutions are

$$
A_{s}^{\circ}(t)=\frac{(i t)^{s}}{s !} \exp \left(\frac{-k^{2} t^{2}}{2 \beta m}\right) \text {. }
$$

Thus we find that while initially the total vector $R_{\stackrel{\mathrm{k}}{\circ}}^{\circ}(t)$ lies wholly along $\Psi_{0}^{\circ}$, this component decreases monotonically in time, while the components along all other $\Psi_{s}^{\circ}$ initially increase, peak in succession, and eventually also vanish.

Finally, using (40) and (42), we find for the complete expansion of $R_{\overrightarrow{\mathrm{k}}}^{\circ}(t)$

$$
R_{\stackrel{\mathrm{k}}{\circ}}(t)=\sum_{j=1}^{N} \exp \left[-i \overrightarrow{\mathrm{k}} \cdot\left(\overrightarrow{\mathrm{r}}_{j}+\frac{\overrightarrow{\mathrm{p}}_{j} t}{m}\right)\right]-N \delta_{\overrightarrow{\mathrm{k}}, 0},
$$

where we have used the generating function of the Hermite polynomials. Obviously this elementary result could have been much more easily obtained directly had it been our main goal.

We have in Eq. (42), for $s=0$, the well-known form of $G_{\mathrm{k}}^{\circ}(t)$ for ideal gases ${ }^{1,7}$ :

$$
A_{0}^{\circ}(t) \equiv G_{\overrightarrow{\mathrm{k}}}^{\circ}(t) / G_{\overrightarrow{\mathrm{k}}}^{\circ}(0)=\exp \left(-k^{2} t^{2} / 2 \beta m\right) .
$$

The Laplace transform of this is

$$
\begin{aligned}
a_{0}^{\circ}(z)= & \left(\pi \beta m / 2 k^{2}\right)^{1 / 2}\left\{1-\operatorname{erf}\left[z\left(\beta m / 2 k^{2}\right)^{1 / 2}\right]\right\} \\
& \times \exp \left(\beta m z^{2} / 2 k^{2}\right),
\end{aligned}
$$

which, with Eq. (29), serves to define $\Re_{\overrightarrow{\mathrm{k}}}^{\circ}(z)$, to be used in the sequel.

\section{APPROXIMATE MEMORY FUNCTION}

In this section we seek to approximate the memory function $K_{\overrightarrow{\mathrm{k}}}(t)$ for the general case of an interacting system. It will be convenient to work temporarily with the finite determinants of Eq. (27), passing to the limit $S \rightarrow \infty$ at the end.

The approach is essentially that of a perturbation expansion, with the ideal gas as the unperturbed system. We have, from Eqs. (30) and (27),

$$
\mathfrak{K}_{\overrightarrow{\mathrm{k}}}^{(S)}(z) \equiv i \nu_{0} D_{2}^{(S)}(z) / D_{1}^{(S)}(z)
$$

where the determinants contain $\nu_{s}, s=1,2, \ldots$, $S-1$, which we write in the form

$$
\nu_{s}=\nu_{s}^{\circ}+\delta_{s} .
$$

An expansion of the determinants in powers of the $\delta_{s}$ can be obtained by using special properties of the $\mathfrak{D}_{s}^{(m)}(z)$. These determinants, called continuants by Muir and Metzler, ${ }^{8}$ satisfy the decomposition rule $^{8}$ 


$$
D_{s}^{(m)}(z)=D_{s}^{(l)}(z) D_{l+1}^{(m)}-\nu_{l} D_{s}^{(l-1)}(z) D_{l+2}^{(m)}(z)
$$

for $s \leq l<m$, where we have incorporated the convention

$$
D_{l+1}^{(l)}(z)=1 \text {. }
$$

Define now a hybrid determinant $\mathfrak{D}_{s ; l}^{(m)}(z)$ wherein the elements $\nu_{i}$ are the ideal gas $\nu_{i}^{\circ}$ for $s \leq i \leq l$ and the complete $\nu_{i}$ for $l<i<m$. Then choosing $l=s$ in Eq. (48), we write

$$
\begin{aligned}
D_{s}^{(m)}(z) & =\mathbb{D}_{s}^{(s)}(z) D_{s+1}^{(m)}(z)-\left(\nu_{s}^{\circ}+\delta_{s}\right) D_{s}^{(s-1)}(z) D_{s+2}^{(m)}(z) \\
& =D_{s ; s}^{(m)}(z)-\delta_{s} D_{s}^{\circ(s-1)}(z) D_{s+2}^{(m)}(z) . \quad(50)
\end{aligned}
$$

The same operations, with the choice $l=s+1$, may now be performed on the hybrid determinant of (50), giving

$$
\begin{aligned}
D_{s}^{(m)}(z)= & D_{s ; s}^{(s+1)}(z) \mathscr{D}_{s+2 ; s}^{(m)}(z) \\
& -\left(\nu_{s+1}^{\circ}+\delta_{s+1}\right) D_{s ; s}^{(s)}(z) D_{s+3 ; s}^{(m)}(z) \\
& -\delta_{s} D_{s}^{\circ(s-1)}(z) D_{s+2}^{(m)}(z) \\
= & D_{s ; s+1}^{(m)}(z)-\sum_{i=s}^{s+1} D_{s}^{\circ(i-1)}(z) D_{i+2}^{(m)}(z) \delta_{i} .
\end{aligned}
$$

Continuing in this way successively through $l=m$ -1 , we obtain a discrete analog of an integral equation for $\mathfrak{D}_{s}^{(m)}(z)$ :

$D_{s}^{(m)}(z)=D_{s}^{\circ(m)}(z)-\sum_{i=s}^{m-1} D_{s}^{\circ(i-1)}(z) D_{i+2}^{(m)}(z) \delta_{i}$.

In the above the indices of the determinants have been simplified as the nature of the elements permitted; the superscript degree mark identifies, of course, the determinants containing only ideal gas $\nu_{s}^{\circ}$.

Equation (52) may be solved iteratively, yielding finally for $\mathcal{K}_{\overrightarrow{\mathrm{k}}}(z)$, after passage to the limit $S \rightarrow \infty$,

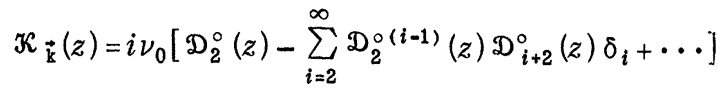

$$
\begin{aligned}
& \times\left[D_{1}^{\circ}(z)-\sum_{i=1}^{\infty} D_{1}^{\circ(i-1)}(z) D_{i+2}^{\circ}(z) \delta_{i}+\cdots\right]^{-1} .
\end{aligned}
$$

Equation (53) is now in a form suitable for approximation. By simply neglecting all $\delta_{s}$ (except $\delta_{0}$, contained in $\nu_{0}$ ) we obtain the lowest approximation,

$$
\mathscr{K}_{\overrightarrow{\mathrm{k}}}(z)=\left(\nu_{0} / \nu_{0}^{\circ}\right) \mathcal{K}_{\mathrm{k}}^{\circ}(z) .
$$

It is shown in Appendix $\mathrm{C}$ that this result leads to a form of the density fluctuation function derived by Nelkin and Ranganathan ${ }^{9}$ from the linearized Vlasov equation. Equation (54) gives the memory function $K_{\overrightarrow{\mathrm{k}}}(t)$ exactly at $t=0$ but treats its time evolution as that of an ideal gas, thus becoming less and less appropriate as time increases.

The next approximation is clearly to neglect all but $\delta_{0}$ and $\delta_{1}$. Then Eq. (53) becomes

$$
\begin{aligned}
\mathcal{K}_{\overrightarrow{\mathrm{k}}}(z) & =\frac{i \nu_{0}{D_{2}^{\circ}}^{\circ}(z)}{\mathscr{D}_{1}^{\circ}(z)-D_{3}^{\circ}(z) \delta_{1}} \\
& =\frac{i \nu_{0} \nu_{1}^{\circ} D_{2}^{\circ}(z)}{\nu_{1}^{\circ} D_{1}^{\circ}(z)-\left[i z D_{2}^{\circ}(z)-i_{1}^{\circ}(z)\right] \delta_{1}} \\
& =\frac{\nu_{0} \nu_{1}^{\circ} \Re_{\overrightarrow{\mathrm{k}}}^{\circ}(z)}{\nu_{0}^{\circ} \nu_{1}-z \delta_{1} \mathfrak{K}_{\overrightarrow{\mathrm{k}}}^{\circ}(z)},
\end{aligned}
$$

where $(48)$ has been used to eliminate $D_{3}^{\circ}(z)$. Note that for large $z$, using the asymptotic form of $\varkappa_{\overrightarrow{\mathrm{k}}}^{\circ}$ $(z)$ in the denominator, one can write

$$
\varkappa_{\overrightarrow{\mathrm{k}}}(z) \sim \frac{\nu_{0} \nu_{1}^{\circ} \mathfrak{K}_{\overrightarrow{\mathrm{k}}}^{\circ}(z)}{\nu_{0}^{\circ} \nu_{1}-\nu_{0}^{\circ} \delta_{1}}=\frac{\nu_{0}}{\nu_{0}^{\circ}} \Re_{\overrightarrow{\mathrm{k}}}^{\circ}(z), \quad z \rightarrow \infty .
$$

That is, just as for the first approximation, the memory function from Eq. (55) is initially exact and evolves as for a free gas for short times.

For long times, ${ }^{10} K_{\overrightarrow{\mathrm{k}}}(t)$ from Eq. (55) will show an exponential decay, with the exponent determined by the root of

$$
\nu_{1} \mathfrak{D}_{1}^{\circ}(z)-i z D_{2}^{\circ}(z)=0
$$

with smallest real part. Exponentially decaying memory functions have been studied by Berne, Boon, and Rice. ${ }^{11}$

A general test of the usefulness of the approximation in Eq. (55) must await a numerical calculation. This is left for a later paper.

\section{APPENDIX A: MATRIX ELEMENTS $\left\langle\Psi_{s}^{*} L \Psi_{n}\right\rangle$}

In order to specify completely the equation of motion of the $A_{s}(t)$, we need to know the effect of operating on a phase function $\Psi_{n}$ with the Liouville operator $L$. From Eqs. (13) we have immediately

$$
\begin{aligned}
& L \Psi_{0}=L R_{\overrightarrow{\mathrm{k}}}(0), \\
& L \Psi_{n}=L^{n+1} R_{\overrightarrow{\mathrm{k}}}(0)-\sum_{i=0}^{n-1} \frac{\left\langle\Psi_{i}^{*} L^{n} R_{\overrightarrow{\mathrm{k}}}(0)\right\rangle}{\left\langle\Psi_{i}^{*} \Psi_{i}\right\rangle} L \Psi_{i},
\end{aligned}
$$

or, using (13) again in the right-hand side,

$$
\begin{aligned}
L \Psi_{0} & =\Psi_{1}+\frac{\left\langle\Psi_{0}^{*} L R_{\overrightarrow{\mathrm{k}}}(0)\right\rangle}{\left\langle\Psi_{0}^{*} \Psi_{0}\right\rangle} \Psi_{0}, \\
L \Psi_{n} & =\Psi_{n+1}+\sum_{i=0}^{n} \frac{\left\langle\Psi_{i}^{*} L^{n+1} R_{\overrightarrow{\mathrm{k}}}(0)\right\rangle}{\left\langle\Psi_{i}^{*} \Psi_{i}\right\rangle} \Psi_{i} \\
& -\sum_{i=0}^{n-1} \frac{\left\langle\Psi_{i}^{*} L^{n} R_{\overrightarrow{\mathrm{k}}}(0)\right\rangle}{\left\langle\Psi_{i}^{*} \Psi_{i}\right\rangle} L \Psi_{i} .
\end{aligned}
$$

This provides a set of equations from which the functions $L \Psi_{n}$ could be calculated successively. Note that $L$ expands $\Psi_{n}$ onto the finite subset $\left\{\Psi_{0}, \cdots, \Psi_{n+1}\right\}$. We now define for convenience a matrix $\Gamma_{i n}$ such that

$$
L \Psi_{n}=\Psi_{n+1}+\sum_{i=0}^{n} \Gamma_{i n} \Psi_{i}
$$


For $s \leq n$, it follows that

$$
\begin{aligned}
\left\langle\Psi_{s}^{*} L \Psi_{n}\right\rangle & =\Gamma_{s n}\left\langle\Psi_{s}^{*} \Psi_{s}\right\rangle \\
& =\left\langle\left(L \Psi_{s}\right)^{*} \Psi_{n}\right\rangle \\
& =\left\langle\left\{\Psi_{s+1}^{*}+\sum_{i=0}^{s} \Gamma_{i s} \Psi_{i}^{*}\right\} \Psi_{n}\right\rangle \\
& =\left\langle\Psi_{s+1}^{*} \Psi_{s+1}\right\rangle \delta_{s+1, n}+\Gamma_{n n}\left\langle\Psi_{s}^{*} \Psi_{s}\right\rangle \delta_{s, n}
\end{aligned}
$$

and hence that

$$
\Gamma_{s n}=\frac{\left\langle\Psi_{s+1}^{*} \Psi_{s+1}\right\rangle}{\left\langle\Psi_{s}^{*} \Psi_{s}\right\rangle} \delta_{s+1, n}+\Gamma_{n n} \delta_{s n}
$$

where we have used the Hermitian property of $L$ in (A4). Explicit expressions for the diagonal elements $\Gamma_{n n}$ may be obtained from (A2). One finds

$$
\begin{aligned}
& \Gamma_{00}=\frac{\left\langle\Psi_{0}^{*} L R_{\overrightarrow{\mathrm{k}}}(0)\right\rangle}{\left\langle\Psi_{0}^{*} \Psi_{0}\right\rangle}, \\
& \Gamma_{n n}=\frac{\left\langle\Psi_{n}^{*} L^{n+1} R_{\overrightarrow{\mathrm{k}}}(0)\right\rangle}{\left\langle\Psi_{n}^{*} \Psi_{n}\right\rangle}-\frac{\left\langle\Psi_{n-1}^{*} L^{n} R_{\overrightarrow{\mathrm{k}}}(0)\right\rangle}{\left\langle\Psi_{n-1}^{*} \Psi_{n-1}\right\rangle}, n>0 .
\end{aligned}
$$

We now note a general rule of these phase averages: Because of the symmetry of the momentum weight function, any integrand with odd powers of the momenta will yield a zero contribution. Thus it follows immediately that $\Gamma_{00}$ vanishes. In general, this rule can be used to establish the vanishing of any phase integral that contains an odd power of the operator $L$. Then, referring to Eqs. (13), we see that because $\Gamma_{00}$ vanishes, $\Psi_{1}$ contains $L$ only linearly. Hence, $\Psi_{2}$ will contain only even powers of $L$, since the coefficient of the linear term vanishes. Continuing in this way, it is apparent that $\Psi_{n}$ will contain only even or odd powers of $L$ accordingly as $n$ is even or odd. It then follows from (A6) that $\Gamma_{n n}$ vanishes for all $n$.

Summing up, we have shown that the only nonvanishing elements of $\Gamma_{i n}$ are those having $i=n-1$, so that, from Eq. (A3),

$$
\begin{aligned}
& L \Psi_{0}=\Psi_{1}, \\
& L \Psi_{n}=\Psi_{n+1}+\nu_{n-1} \Psi_{n-1}, \quad n>0
\end{aligned}
$$

where

$$
\nu_{n} \equiv \Gamma_{n, n+1}=\left\langle\Psi_{n+1}^{*} \Psi_{n+1}\right\rangle /\left\langle\Psi_{n}^{*} \Psi_{n}\right\rangle
$$

\section{APPENDIX B: COEFFICIENTS $\nu_{s}$}

The proof of Eqs. (39) and (40) for the ideal gas proceeds by alternate steps. It is readily verified that the first two functions $\Psi_{s}^{\circ}$ can be written in the form

$$
\begin{aligned}
& \Psi_{0}^{\circ}=\sum_{j=1}^{N} e^{-i \overrightarrow{\mathrm{k}} \cdot \overrightarrow{\mathrm{r}}_{j}} H_{0}\left[\left(\frac{\beta}{m}\right)^{1 / 2} \hat{k} \cdot \overrightarrow{\mathrm{p}}_{j}\right]-N \delta_{\overrightarrow{\mathrm{k}}, 0}, \\
& \Psi_{1}^{\circ}=-\left(\frac{k^{2}}{\beta m}\right)^{1 / 2} \sum_{j=1}^{N} e^{-i \overrightarrow{\mathrm{k}} \cdot \overrightarrow{\mathrm{r}}_{j}} H_{1}\left[\left(\frac{\beta}{m}\right)^{1 / 2} \hat{k} \cdot \overrightarrow{\mathrm{p}}_{j}\right]
\end{aligned}
$$

where the $H_{n}(x)$ are Hermite polynomials ${ }^{12}$ and $\hat{k}$ is a unit vector in the direction of $\vec{k}$. It follows that

$$
\begin{aligned}
& \left\langle\Psi_{0}^{\circ *} \Psi_{0}^{\circ}\right\rangle=N\left(1-\delta_{\overrightarrow{\mathrm{k}}, 0}\right), \\
& \left\langle\Psi_{1}^{\circ} * \Psi_{1}^{\circ}\right\rangle=N k^{2} / \beta m,
\end{aligned}
$$

whence, using (A8),

$$
\nu_{0}^{\circ}=k^{2} / \beta m \text {. }
$$

With the use of (A7) in the form

$$
\Psi_{2}^{\circ}=L_{0} \Psi^{\circ}-\nu_{0}^{\circ} \Psi_{0}^{\circ}
$$

and the recurrence relation for Hermite polynomials, one can show that $\Psi_{2}^{\circ}$ may also be written in the form

$\Psi_{s}^{\circ}=(-1)^{s}\left(\frac{k^{2}}{\beta m}\right)^{s / 2} \sum_{j=1}^{N} e^{-i \overrightarrow{\mathrm{k}} \cdot \overrightarrow{\mathrm{r}}_{j}} H_{s}\left[\left(\frac{\beta}{m}\right)^{\dagger / 2} \hat{k} \cdot \overrightarrow{\mathrm{p}}_{j}\right]$,

and hence, again using (A8), that

$$
\nu_{1}^{\circ}=2 k^{2} / \beta m \text {. }
$$

By an obvious proof using mathematical induction with Eq. (A7) and the recurrence relation of the Hermite polynomials, it follows that all further $\Psi_{s}^{\circ}$ can be written in the form of Eq. (B7), while correspondingly the coefficients $\nu_{s}^{\circ}$ are

$$
\nu_{s}^{\circ}=(s+1) k^{2} / \beta m \text {. }
$$

For the general interacting case we display here only the first two coefficients $\nu_{j}$. By a straightforward calculation, it is found that

$$
\begin{aligned}
& \nu_{0}=\nu_{0}^{\circ}-\left(n k^{2} / \beta m\right) C_{\overrightarrow{\mathrm{k}}}, \\
& \nu_{1}=\nu_{1}^{\circ}+\frac{n k^{2}}{\beta m} C_{\overrightarrow{\mathrm{k}}}+\frac{n}{m} \int d \overrightarrow{\mathrm{r}}\left(1-e^{i \overrightarrow{\mathrm{r}} \cdot \overrightarrow{\mathrm{r}}}\right) g(r)(\hat{k} \cdot \nabla)^{2} \phi(r) .
\end{aligned}
$$

In obtaining (B11), we have assumed the intermolecular potential is composed of binary interactions only:

$$
U=\sum_{i<j} \phi_{i j}
$$

These coefficients involve the second and fourth moments of $G_{\overrightarrow{\mathrm{k}}}(t)$, which were first given by de Gennes. ${ }^{13}$

\section{APPENDIX C: EQUATION (54) AND NELKIN-RANGANATHAN SOLUTION (NR)}

In this appendix we wish to calculate explicitly the time Fourier transform of $G_{\overrightarrow{\mathrm{k}}}(t)$,

$$
S_{\overrightarrow{\mathrm{k}}}(\omega)=(2 \pi)^{-1} \int_{-\infty}^{\infty} n G_{\overrightarrow{\mathrm{k}}}(t) e^{-i \omega t} d t,
$$

using the approximation of Eq. (54). Because of the symmetry in time of $G_{\overrightarrow{\mathrm{k}}}(t)$, Eq. (C1) may be written 


$$
\begin{aligned}
S_{\overrightarrow{\mathbf{k}}}(\omega) & =\pi^{-1} \operatorname{Re}\left[\int_{0}^{\infty} n G_{\overrightarrow{\mathrm{k}}}(t) e^{-i \omega t} d t\right] \\
& =\pi^{-1} \operatorname{Re}\left[n \oint_{\overrightarrow{\mathbf{k}}}(i \omega)\right] .
\end{aligned}
$$

Now, using Eq. (29) for both the ideal gas and the general case, one obtains from Eq. (54)

$$
a_{0}(z)=\frac{a_{0}^{\circ}(z)}{1+\left(1-\nu_{0} / \nu_{0}^{\circ}\right)\left[z Q_{0}^{\circ}(z)-1\right]},
$$

or

$$
\mathcal{S}_{\overrightarrow{\mathrm{k}}}(z)=\frac{a_{0}^{\circ}(z) G_{\overrightarrow{\mathrm{k}}}(0)}{1+n C_{\overrightarrow{\mathrm{k}}}\left\{z Q_{0}^{\circ}(z)-1\right\}}
$$

where we have used Eqs. (17), (B9), and (B10). With the mathematical result that

$$
\operatorname{erf}(i x)=(2 i / \sqrt{ } \pi) \int_{0}^{x} d s e^{s^{2}},
$$

we obtain from Eq. (45)

$$
a_{0}^{\circ}(i \omega)=\left(\beta m / 2 k^{2}\right)^{1 / 2}[A(x)-i B(x)],
$$
having defined, following NR,

$$
\begin{aligned}
x & =\left(\beta m / 2 k^{2}\right)^{1 / 2} \omega, \\
A(x) & =\sqrt{ } \pi e^{-x^{2}}, \\
B(x) & =2 e^{-x^{2}} \int_{0}^{x} e^{s^{2}} d s .
\end{aligned}
$$

Thus, after some simplification, we get

$$
S_{\overrightarrow{\mathrm{k}}}(\omega)=\frac{(2 \pi)^{-1}\left(2 \beta m / k^{2}\right)^{1 / 2} A(x)}{\left[\left(1+n G_{\overrightarrow{\mathrm{k}}}\right)^{-1}+n C_{\overrightarrow{\mathrm{k}}} x B(x)\right]^{2}+\left[n C_{\overrightarrow{\mathrm{k}}} x A(x)\right]^{2}},
$$

which is the result obtained by Nelkin and Ranganathan ${ }^{9}$ from the linearized Vlasov equation, with an additional factor of $2 \pi$ from the definition.
* Supported in part by the North Carolina Engineering Foundation.

${ }^{1}$ L. Van Hove, Phys. Rev. 95, 249 (1954).

${ }^{2} \mathrm{R}$. Zwanzig, in Lectures in Theoretical Physics, edited by W. E. Brittin, W. B. Downs, and J. Downs (Interscience, New York, 1961), Vol. 3, p. 135.

${ }^{3}$ Much of this formal development amounts to a transcription of the classical theory of orthogonal polynomials with $\partial / \partial t$ replacing $x$. See, e.g., G. Szegö, Orthogonal Polynomials (American Mathematical Society Colloquium Publications, New York, 1939), Vol. 23.

${ }^{4}$ H. Mori, Progr. Theoret. Phys. (Kyoto) 34, 399 (1965).

${ }^{5}$ This function is actually a trivial modification of the Van Hove $G(\vec{r}, t)$.

${ }^{6}$ J. K. Percus and G. J. Yevick, Phys. Fluids $\underline{11}$, 2050 (1968). For another derivation see F. Lado, ibid. 13, 1396 (1970).

'P. A. Egelstaff, An Introduction to the Liquid State
(Academic, New York, 1967), Chap. 9.

${ }^{8} \mathrm{~T}$. Muir and W. H. Metzler, A Treatise on the Theory of Determinants (Dover, New York, 1960), Chap. 13.

${ }^{9}$ M. Nelkin and S. Ranganathan, Phys. Rev. 164, 222 (1967); see also P. Ortoleva and M. Nelkin, ibid. 181, 429 (1969).

${ }^{10}$ The initial correlations actually are inadequate to describe the long-time behavior of the correlation functions, and additional assumptions must be used, as in Ref. 11 . This is especially noticeable in the case of hard cores, where the integrals defining the $\nu_{n}$ are divergent for $n$ $>0$.

${ }^{11}$ B. J. Berne, J. P. Boon, and S. A. Rice, J. Chem. Phys. 4드, 1086 (1966).

${ }^{12}$ D. Jackson, Fourier Series and Orthogonal Polynomials (The Mathematical Association of America, Oberlin, Ohio, 1957).

${ }^{13}$ P. G. de Gennes, Physica 25,825 (1959). 